DOE/ER/14090--3

DE93 001838

\title{
PULSE PROPAGATION IN INHOMOGENEOUS OPTICAL FIBERS
}

\author{
Progress Report \\ $9 / 15 / 91-9 / 14 / 92$ \\ Curtis R. Menyuk \\ Department of Electrical Engineering \\ University of Maryland \\ Baltimore, MD 21228-5398
}

\author{
May 29,1992 \\ Prepared for the US Department of Energy \\ under grant no. DE-FG05-89ER14090
}

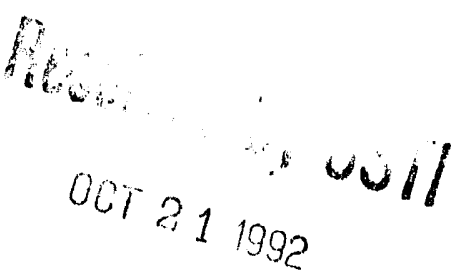

\section{DISCLAIMER}

This report was prepared as an account of work sponsored by an agency of the United States Government. Neither the United States Gnvernment nor any agency thereof, nor any of their employees, makes any warranty, express or implied, or assumes any legal liability or responsibility for the accuracy, completeness, or usefulness of any information, apparatus, product, or process disclosed, or represents that its use would not infringe privately owned rights. Reference herein to any specific commercial product, process, or service by trade name, trademark, manufacturer, or otherwise does not necessarily constitute or imply its endorsement, recommendation, or favoring by the United States Government or any agency thereof. The views and opinions of authors expressed herein do not necessarily state or reflect those of the United States Government or any agency thereof.

\section{MASTER}

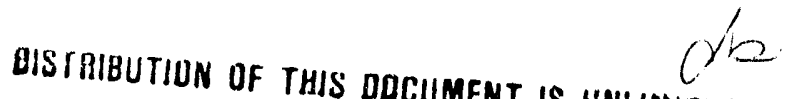




\section{PULSE PROPAGATION IN INHOMOGENEOUS OPTICAL FIBERS}

\section{Summary of Accomplishments}

A little more than two and one-half years into the period of our present grant "Pulse propagation in inhomogeneous optical fibers," we can point with pride to a strong record of accomplishment. These accomplishments include one M.S. thesis, ten publications which have already appeared in print and two more which have been submitted, as well as eighteen conference presentations, including one invited presentation, and one paper which will appear in a workshop proceedings. A complete record of our publications and presentations may be found in Sec. II.D. Far more important than the quantity of publications, however, is the impact of our work on the scientific and engineering communities. The area in which our work has been primarily focused, soliton propagation in optical fibers, is now generally accepted to be the method of choice for trans-oceanic communication at bit rates exceeding 5 Gbits/sec. ${ }^{1,2}$ The situation has substantially changed from two years ago when the use of solitons for trans-oceanic communication was still considered questionable. Our work contributed to this development in two important respects. First, our original work on soliton robustness when fiber birefringence is taken into account ${ }^{3,4}$ led directly to a recent proposal to use polarization division multiplexing. ${ }^{5}$ This technique is only possible with solitons, not linear pulses, and will lead to at least a doubling of the bit rate. In this work, we have interacted closely with Dr. Linn Mollenauer of AT\&T, who is the principal experimentalist working on this subject. Second, the computer codes which we originally wrote to simulate nonlinear pulse propagation in optical fibers have been extensively used, after some modification, by members of the submarine lightsystems group at AT\&T to compare a number of linear and nonlinear schemes for trans-oceanic lightwave communication and have thus played an important role in this group's planning for the future. Indeed, the support from the Department of Energy has made it possible for us to make these simulation codes available at no cost to all qualified users, and versions of our simulation codes are now being used by researchers at Harvard, the University of Colorado, and the Naval Research Laboratory. Researchers from MIT and the University of Michigan have relied on the published algorithms in the design of their own computer codes.

The scope of our work has included significantly more than solitons for trans-oceanic communications. Our work has included a successful collaboration with Dr. Mohammed Islam of AT\&T, who is carrying out switching experiments using optical fiber solitons. This collaboration has been both experimental and theorctical. Our work has included collaborations with Irl Duling at the the Naval Research Laboratory and scientists at AT\&T who are looking at soliton sources, both in the long duration, 50 ps regime of interest for long-distance communications and in the short duration, sub-picosecond regime of interest for switching and probing of solid state devices. We have also interacted with 
Dr. Jon Sauer at the University of Colorado ERC, who is looking at the possibility of using wavelength division multiplexed solitons in medium-range, $20 \mathrm{~km}--100 \mathrm{~km}$, computer interconnects.

The approach which we have followed of interacting with leading experimental researchers, sometimes collaborating while sometimes pursuing our own approaches, has allowed us to achieve significant recognition in a very device-oriented community. These recognitions include: 1) A standing invitation from Dr. Islam to accept highly motivated and capable students into his laboratory to participate in experiments. Mr. Chen of our research group has already availed himself of this opportunity. 2) Dr. Menyuk, the principal investigator was invited to lecture in a summer school in Corsica. The other U.S. lecturers from universities were Dr. Desurvire of Columbia, the inventor of Er-doped fiber amplifiers, Dr. Fejer of Stanford, the inventor of quasi-phase-matched second harmonic generation, and Dr. Bloembergen of Harvard, a Nobel prize winner. 3) Dr. Menyuk was an invited speaker at the most recent annual meeting of the Optical Society of America, where he was invited by Dr. Mollenauer to speak at a symposium on long-distance soliton communications - the only speaker who had never held a position at AT\&T.

We have broadened the scope of our originally proposed work, with the agreement of our technical monitor, Dr. Manley, to include inhomogeneities in solid state waveguides. Our principal experimental collaborator in this effort has been Dr. Y. J. Chen of UMBC, and we have recently begun to interact with the group of Dr. George Simonis at Harry Diamond Laboratories. With the codes we have developed, we are now poised to begin an effort on quasi-phase-matched second harmonic generation. In this effort, we intend to interact with Dr. John Bierlein of Dupont. The technological motivation for studying second harmonic generation is that it allows one to double infrared wavelengths into the visible. Laser diodes which are powerful enough to write on compact disks exist at present at infrared wavelengths but do not exist at visible wavelengths. Second harmonic generation offers the possibility of quadrupling the information density which can be written to compact disks. A fundamental difficulty is that dispersion in waveguides makes phasematching difficult. Fejer and co-workers ${ }^{6}$ at Stanford proposed and demonstrated that quasi-phase-matching can be achieved by periodically changing the sign of the nonlinearity in the guide at the difference wavelength between the fundamental and $2 \times$ the second harmonic wavelength. Due to material difficulties, the Stanford group has not achieved the efficiencies necessary for commercial applications. Recently, however, these difficulties were circumvented by Bierlein and co-workers ${ }^{7}$ at Dupont who have brought this approach to the edge of technological realization. The time is now ripe for careful simulations of the waveguide interactions, both to gain insight into the basic processes which are occuring and to optimize (or more precisely to provide the basic tools for optimization) the geometry for engincering applications. To our knowledge, therc have been no simulations of these waveguides to date. 


\section{Detailed Project Description}

\section{A. Optical Fibers}

Our work on light propagation in optical fibers has been in three principal research areas: 1) randomly varying birefringence in optical fibers, 2) soliton switching in optical fibers, and 3) fiber loop and ring lasers. This work will be described sequentially.

The work on randomly varying birefringence has used a simple model in which the birefringence undergoes a complete randomization at regular intervals, both to determine the robustness of solitons which are in a single polarization state as a function of time at the initial point along the fiber and to determine the strength of the interaction between solitons due to the randomly varying birefringence. In this work, we were very careful to use a physically appropriate ordering of length scales. We stress this point because in some of the work carried out to date on this problem, it is effectively assumed that the coherence length of the birefringence is short compared to the average beat length, ${ }^{8}$ an assumption which makes the problem more tractable analytically but which is not accurate. We found that to lowest order in an appropriate perturbation expansion, the nonlinear Schrödinger equation appears. ${ }^{9}$ This result is of importance from a practical viewpoint as it determines when polarization division multiplexing is practical. We also found that randomly varying birefringence cannot account for the experimentally observed long-range interaction between solitons. ${ }^{10}$

Our collaboration with Dr. Mohammed Islam of AT\&T Bell Laboratories, both experimental and theoretical, has been the focus of much of our work during the past two years. The physical basis of the optical fiber switches being studied by Islam and by us is that when solitons of different polarizations interact in a birefringent optical fiber, their frequencies shift so that their velocities are altered. ${ }^{3,11}$ One soliton is the control pulse and the other is the signal pulse. When the control pulse is present, the time slot in which the signal pulse appears is changed. These switches can be used to construct a Boolean complete set of logic gates, and they can be used in a high information rate ring architecture. ${ }^{12}$ The issues on which we have concentrated include the impact of the Raman effect, ${ }^{13,14}$ schemes to separate the frequency shifting function from the dragging function so that lower latencies and lower switching energies can be simultaneously achieved, ${ }^{15-18}$ and fundamental studies of shadow and radiation generation which limit the effectiveness of the switches. These latter studies are now being prepared for publication. Through these studies, it has become clear that while switching energies of 1 pJ have been achieved, it is at the cost of microsecond latencies. Thus, these switches must be used in ring architectures where the latency does not matter and only a small number of high speed switches need be used. We have also learned how to do timing restoration, an important fcature in any switch.

Our work on ring and loop lasers has focused on two distinct laser systems. The first is a ring laser originally proposed as a soliton source by Mollenauer. ${ }^{19}$ Mollenauer has not yet published his configuration, but he has made the details of its workings and preliminary experimental results available to us. For the laser to produce solitons pulses, it is essential that it mode lock, i.e., the phase relationship of the different frequency components of the 
output must be coherent. When the laser was originally demonstrated, Mollenauer and coworkers assumed that self-phase-modulation in a long fiber length produced mode locking. We showed that the mode locking could not be produced by self-phase-modulation and that some other source was needed. We proposed that mode locking in Mollenauer's configuration was due to nonlinear polarization rotation coupled with polarization selectivity. We also showed that a frequency limiter was necessary to achieve stable laser operation. Finally, we proposed that the laser operation could be optimized by imposing mode locking, either by explicitly using polarization selective elements or by using a GaAs quantum well absorber. This work, which has been submitted for publication, has already impacted on laser design efforts being carried out by two groups at AT\&T, one which is aimed at producing 50 ps pulses for long-distance communication applications, and the other of which is being aimed at produring sub-picosecond pulses for switching and solid-state probing experiments.

The second system on which we have focused is the figure 8 loop laser of Irl Duling. ${ }^{20}$ In this configuration, a loop mirror with assymetric gain is combined with a second, unidirectional loop. The loop mirror contributes the mode locking, and sub-picosecond pulses have been achieved. The laser's operation is somewhat unstable, and our results to date suggest that frequency filtering may be helpful, although the filtering does limit the minimum pulse duration achievable. However, this work is in a very early stage.

\section{B. Solid State Waveguides}

During the grant period, much of our work on solid state waveguides focused on code development. However, this phase of our work has been completed with the creation of a finite element code $\mathrm{e}^{21}$ for determining both mode propagation wavenumbers and profiles in waveguides which are homogeneous in the direction of propagation and with the creation of a beam propagation $\operatorname{code}^{22}$ for determining the wave evolution in cases where the waveguides are inhomogeneous in the direction of propagation. Code development continues and, indeed, we recently wrote a code based on the effective index method ${ }^{23}$ for finding the modes of a waveguide, thus allowing us to use the more accurate but more computationally intensive finite element method for a final refinement of our parameters rather than all the time. However, we are no long focusing on code development. Instead, our focus is now on solving problems of practical interest to the experimental groups with which we interact.

In collaboration with Professor Y. J. Chen of UMBC, we have studied two problems. The first was to determine the effect of a realistic rib waveguide geometry on the propagation charactcristics of a wave in a guide with a quantum well layer and an electrostatic field. By changing the voltage transverse to the guide, one controls the quantum confined Stark effect and, hence, both the loss and phase change due to the quantum well layer. The quantum confined Stark effect has becn well-studied in a planar geometry, ${ }^{24}$ but a planar 
geometry cannot be used in devices such as modulators or interferometers, so that a study of realistic geometries must be made. We found that for the parameters of interest to the experimentalists, the change due to the rib geometry is not large. The most significant change is a large percentage increase in the loss at high voltages, although the overall loss is still small at high voltages. ${ }^{21}$

The second problem was to determine the effect of tip rounding, as opposed to tip blunting, on the radiation loss in a $Y$ junction. Rounding occurs when dry chemical etching is used, as opposed to blunting which occurs when wet chemical etching is used. Professor Chen and his collaborators use the former process. We found that rounding, as opposed to blunting, has surprisingly little effect. We finc that for any reasonable value of rounding and of the point at which the $Y$ junction splits, the rounding leads to little additional radiation. Thus, it is more benign in its effects than blunting.

\section{Code Development}

As our codes have become increasingly used outside our own group, we have paid increasing attention to ensuring that our own codes are mutually compatible, well documented, and reasonably user friendly. Our optical fiber codes have been structured in a modular fashion so that different physics subroutines can be added or removed as requirsd. All our codes have been optimized to run on CRAY supercomputers. We have developed a set of DISSPLA-based graphics routines which allow us to do our initial graphics processing on a CRAY. Most of our post-processing is carried out interactively on Apple computers and HP workstations. We also have MATLAB versions of some of our more rapidly running codes, notably the effective index code, which run rapidly on workstations. These codes are also well-documented.

D. Publications and Presentations

M.S. THESIS

1. P. Subramanian, A Study of Light Propagation in Y Junction Waveguides (M.S. Thesis, August, 1991). 


\section{JOURNAL PUBLICATIONS}

1. C.J. Chen, P.K.A. Wai, and C.R. Menyuk, "A Soliton Switch Using Birefringent Optical Fibers," Optics Lett. 15, 477-479 (1990).

2. M.N. Islam, C.E. Soccolich, C.-J. Chen, K.S. Kim, J.R. Simpson, and U.C. Paek, "AllOptical Inverter With One Picojoule Switching Energy," Electron. Lett. 27, 130-132 (1991).

3. M.N. Islam, C.R. Menyuk, C.-J. Chen, and C.E. Soccolich, "Chirp Mechanisms in Soliton Dragging Logic Gates," Optics Lett. 16, 214-215 (1991).

4. M.N. Islam, C.-J. Chen, and C.E. Soccolich, "All-Optical Time-Domain Chirp Switch," Optics Lett. 16, 484-486 (1991).

5. C.R. Menyuk, M.N. Islam, and J.P. Gordon, "Raman Effect in Birefringent Optical Fibers," Optics Lett. 16, 566-568 (1991).

6. M.N. Islam, C.E. Soccolich, C.-J. Chen, U.-C. Paek, C.M. Schroeder, D.J. DiGiovanni, and J.R. Simpson, "Prechirper to Relax the Timing Restrictions for Soliton-Dragging Logic Gates," Optics Lett. 16, 593-595 (1991).

7. P.K.A. Wai, C.R. Menyuk, and H.H. Chen, "Stability of Solitons in Randomly Varying Birefringent Fibers," Optics Lett. 16, 1231-1233 (1991).

8. C.-J. Chen, C.R. Menyuk, M.N. Islam, and R.H. Stolen, "Numerical Study of the Raman Effect and Its Impact on Soliton Dragging Logic Gates," Optics Lett. 16, 1647-1649 (1991).

9. P.K.A. Wai, C.R. Menyuk, and H.H. Chen, "Effects of Randomly. Varying Birefringence on Soliton Interactions in Optical Fibers," Optics Lett. 16, 1735-1737 (1991).

10. V.R Chinni, C.R. Menyuk, and Y.J. Chen, "Propagation Characteristics of GaAs/ AlGaAs QW Riage Waveguides," IEEE Photon. Tech. Lett. 3, 1120-1123 (1991).

11. C.J. Chen, P.K.A. Wai, and C.R. Menyuk, "Soliton Fiber Ring Laser," Optics Lett. 17, 417-419 (1992).

12. P. Subramanian, P.K.A. Wai, C.R. Menyuk, and R.J. Hawkins, "Effects of Junction Rounding on Transmission Characteristics of Y Junctions," (submitted to IEEE Photonics Technology Letters).

13. Q. Wang, P.K.A. Wai, C.J. Chen, and C.R. Menyuk, "Soliton Shadows in Birefringent Optical Fibers," (submitted to Optics Letters).

\section{PROCEEDING PUBLICATION}

1. C.R. Menyuk, "Why are Solitons Robust in Optical Fibers?" in Guided Wave Nonlinear Optics, edited by D. Ostrowsky and R. Reinisch (Kluwer, to appear).

2. C.R. Menyuk and P.K.A. Wai, "Soliton Propagation in an Optical Fiber With Randomly Varying Birefringence," in Proceedings of the Tenth Symposium on Energy Engineering Science, edited by O.P. Manley (US Gov. Printing Office, Washington, to appear). 


\section{CONFERENCE PRESENTATIONS \\ * indicates invited presentation}

1. C.J. Chen, P.K.A. Wai, and C.R. Menyuk, "All-Optical Switch Using Elliptically Birefringent Single-Mode Fiber," Optical Society of America Annual Meeting, Orlando, FL (Oct. 15-20, 1989), paper TuF6.

2. C.R. Menyuk, J.P. Gordon, and M.N. Islam, "The Raman Effect in Birefringent Optical Fibers," Integrated Photonics Research Topical Meeting, Hilton Head, SC (March 26-28, 1990), paper TuB5.

3. M.N. Islam, C.E. Soccolich, and C.-J. Chen, "All-Optical Time Domain Chirp Switch," Optical Society of America Annual Meeting, Boston, MA (Nov. 4-9, 1990), paper WM2.

4. C.-J. Chen, C.R. Menyuk, M.N. Islam, and R. Stolen, "Numerical Simulation of the Raman Effect in Birefringent Optical Fibers," Optical Socieiy of America Annual Meeting, Boston, MA (Nov. 4-9, 1990), paper WU11.

5. P.K.A. Wai and C. R. Menyuk, "Stability of Solitons in Randomly Varying Birefringent Fibers," Optical Society of America Annual Meeting, Boston, MA (Nov. 4-9, 1990), paper ThY56.

6. M.N. Islam, C.R. Menyuk, C.J. Chen, and C.E. Soccolich, "Chirp Mechanisms in Soliton Dragging Logic Gates, "Integrated Photonics Research Topical Meeting, Monterey, CA (April 9-11, 1991), paper TuA2.

7. M.N. Islam, C.-J. Chen, and C.E. Soccolich, "All-Optical Time Domain Chirp Switches With One Picojoule of Switching Energy," Integrated Photonics Research Topical Meeting; Monterey, CA (April 9-11, 1991), paper TuE1.

8. C.R. Menyuk, "Partial Collisions of Solitons in Birefringent Optical Fibers," Integrated Photonics Research Topical Meeting, Monterey, CA (April 9-11, 1991), paper WF3.

9. M.N. Islam, C.E. Soccolich, C.J. Chen, K.S. Kim, J.R. Simpson, and U.C. Paek, "AllOptical Inverter With 1 pJ Switching Energy," Conference on Lasers and ElectroOptics, Baltimore, MD (May 12-17, 1991), paper CMB3.

10. P.K.A. Wai and C.R. Menyuk, "Effects of Randomly Varying Birefringence in Long Distance Soliton Communication System," Conference on Lasers and Electro-Optics, Baltimore, MD (May 12-17, 1991), paper CWG3.

11. C.-J. Chen, M.N. Islam, C.E. Soccolich, U.-C. Pack, C.M. Schroeder, D.J. DiGiovanni, and J.R. Simpson, "Prechirper to Relax Timing Restrictions for Soliton-Dragging Logic Gates," Conference on Lasers and Electro-Optics, Baltimore, MD (May 12-17, 1991), paper CWG3.

12. P.K.A. Wai, C.R. Menyuk, and H.H. Chen, "Soliton Propagation in a Randorn Medium," III Potsdam - V Kiev 'nternational Workshop on Nonlinear Processes in Physics, Potsdam, NY (Clarkson University, August 1-11, 1991). 
13. C.-J. Chen, P.K.A. Wai, and C.R. Menyuk, "Soliton Fiber Ring Laser Using an Erbium-Doped Fiber Amplifier," Optical Society of America Annual Meeting, San Jose, CA (Nov. 3-8, 1991), paper MT4.

14. I.N. Duling III, C.J. Chen, and C.R. Menyuk, "Propagation of Solitons in a Nonlinear Loop Mirror," Optical Society of America Annucl Meeting, San Jose, CA (Nov. 3-8, 1991), paper MGG4.

15. P.K.A. Wai, C.R. Menyuk, and H.H. Chen, "Effects of Randomly Varying Birefringence on Soliton Interactions in Optical Fibers," Optical Society of America Annual Meeting, San Jose, CA (Nov. 3-8, 1991), paper MRR4.

16. V.R. Chinni, C.R. Menyuk, and Y.J. Chen, "Propagation Characteristics of GaAs/ AlGaAs QW Ridge Waveguides," Optical Society of America Annual Meeting, San Jose, CA (Nov. 3-8, 1991), paper MTT2.

*17. C.R. Menyuk, "Effect of Optical Fiber Birefringence on Long-Distance Soliton Transmission," Optical Society of America Annual Meeting, San Jose, CA (Nov. 3-8, 1991), paper TuA3.

18. P. Subramanian, P.K.A. Wai, C.R. Menyuk, and R.J. Hawkins, "Effect of Junction Rounding on the Propagation Characteristics of Y-Junction Waveguides," Optical Society of America Annual Meeting, San Jose, CA (Nov. 3-8, 1991), paper TuII1.

19. V.R. Chinni, C.R. Menyuk, and Y.J. Chen, "Propagation Characteristics of GaAs/ AlGaAs QW Ridge Wavegides," S.P.I.E. Conference on Emerging Optoelectronic Technologies, Bangalore, India (Dec. 16-21, 1991).

20. C.R. Menyuk, "A Recipe For Soliton Robustness in Optical Fibers," Integrated Photonics Research Topical Meeting, New Orleans, LA (April 13-16, 1992), paper TuI4.

*21. C.R. Menyuk, "Soliton Propagation in an Optical Fiber With Randomly Varying Birefringence," Tenth Symposium on Energy Engineering Science, Argonne, IL (May 11-13, 1992). 


\section{References}

1. J.J. Keller, "Defying Boss's Orders Pays Off for Physicist and His Firm, AT\&T," The Wall Street Journal (June 25, 1991).

2. L.F. Mollenauer, J.P. Gordon, and S.G. Evangelides, Laser Focus World, "Multigigabit soliton transmissions traverse ultralong distances," Laser Focus World (November, 1991), p. 159.

3. C.R. Menyuk, Opt. Lett. 12, 614 (1987); J. Opt. Soc. Am. B 5, 392 (1988).

4. L.F. Mollenauer, K. Smith, J.P. Gordon, and C.R. Menyuk, Opt. Lett. 14, 1219 (1989).

5. S.G. Evangelides, L.F. Mollenauer, and J.P. Gordon, "Polarization multiplexing with solitons," J. Lightwave Tech. (to be published).

6. M.M. Fejer, G.A. Magel, D.H. Jundt, and R.L. Byer, "Quasi-phase-matched second harmonic generation: tuning and tolerances," IEEE J. Quantum Electron. (submitted for publication); E.J. Lim, S. Matsumoto, and M.M. Fejer, Appl. Phys. Lett. 57, 2294 (1990); E.J. Lim, M.M. Fejer, R.L. Byer, and W.J. Kozlovsky, Electron. Lett. 25, 731 (1989); E.J. Lim, M.M. Fejer, and R.L. Byer, Electron. Lett. 25, 174 (1989).

7. J.D. Bierlein, "Second order nonlinear optical processes in $\mathrm{KTiOPO}_{4}$ waveguides," in Guided Wave Nonlinear Optics, edited by D. Ostrowsky and R. Reinisch (Kluwer, to appear); C.J. van der Poel, J.D. Bierlein, J.B. Brown, and S. Colak, Appl. Phys. Lett. 57, 2074 (1990); J.D. Bierlein, D.B. Laubacher, J.B. Brown, and C.J. van der Poel, Appl. Phys. Lett. 56, 1725 (1990); J.D. Bierlein and H. Vanherzeele, J. Opt. Soc. Am. B 6, 622 (1989).

8. T. Ueda and W.L. Kath, "Dynamics of optical pulses in randomly birefringent fibers," Physica D (accepted for publication).

9. P.K.A. Wai, C.R. Menyuk, and H.H. Chen, Optics Lett. 16,1231 (1991).

10. P.K.A. Wai, C.R. Menyuk, and H.H. Chen, Optics Lett. 16, 1735 (1991).

11. M.N. Islam, Opt. Lett. 14, 1257 (1989); 15, 417 (1990); M.N. Islam, C.E. Soccolich, and D.A.B. Miller, Opt. Lett. 15, 909 (1990).

12. J.R. Sauer, M.N. Islam, and S.P. Dijaili, " $\Lambda$ soliton ring network," IEEE J. Quantum Electron. (accepted for publication).

13. C.R. Menyuk, M.N. Islam, and J.P. Gordon, Opt. Lett. 16, 566 (1991).

14. C.-J. Chen, C.R. Menyuk, M.N. Islam, and R.H. Stolen, Opt. Lett. 16, 1647 (1991).

15. M.N. Islam, C.E. Soccolich, C.-J. Chen, K.S. Kim, J.R. Simpson, and U.C. Pack, Electron. Lett. 27, 130 (1991).

16. M.N. Islam, C.R. Menyuk, C.-J. Chen, and C.E. Soccolich, Opt. Lett. 16, 214 (1991).

17. M.N. Islam, C.-J. Chen, and C.E. Soccolich, Opt. Lett. 16, 484-486 (1991).

18. M.N. Islam, C.E. Soccolich, C.-J. Chen, U. C. Pack, C.M. Schrocder, D.J. DiGiovanni, and J.R. Simpson, Opt. Lett. 16, 593 (1991). 
19. While this work is unpublished, Dr. Mollenauer has presented this configuration in his lecture as one of the 1990-1991 IEEE-LEOS travelling lecturers.

20. I.N. Duling III, Electron. Lett. 27, 544 (1991); Opt. Lett. 16, 539 (1991).

21. V.R. Chinni, C.R. Menyuk, and Y.J. Chen, "Propagation Characteristics of GaAs/ AlGaAs QW Ridge Waveguides," IEEE Photon. Tech. Lett. 3, 1120-1123 (1991).

22. P. Subramanian, P.K.A. Wai, C.R. Menyuk, and R.J. Hawkins, "Effects of Junction Rounding on Transmission Characteristics of Y Junctions," IEEE Photonics Lett. (submitted for publication).

23. The approach we use is described by K.-H. Schlereth and M. Tacke, IEEE J. Quantum Electron. QE-26, 627 (1990).

24. T. H. Wood, Appl. Phys. Lett. 48, 1413 (1986). 

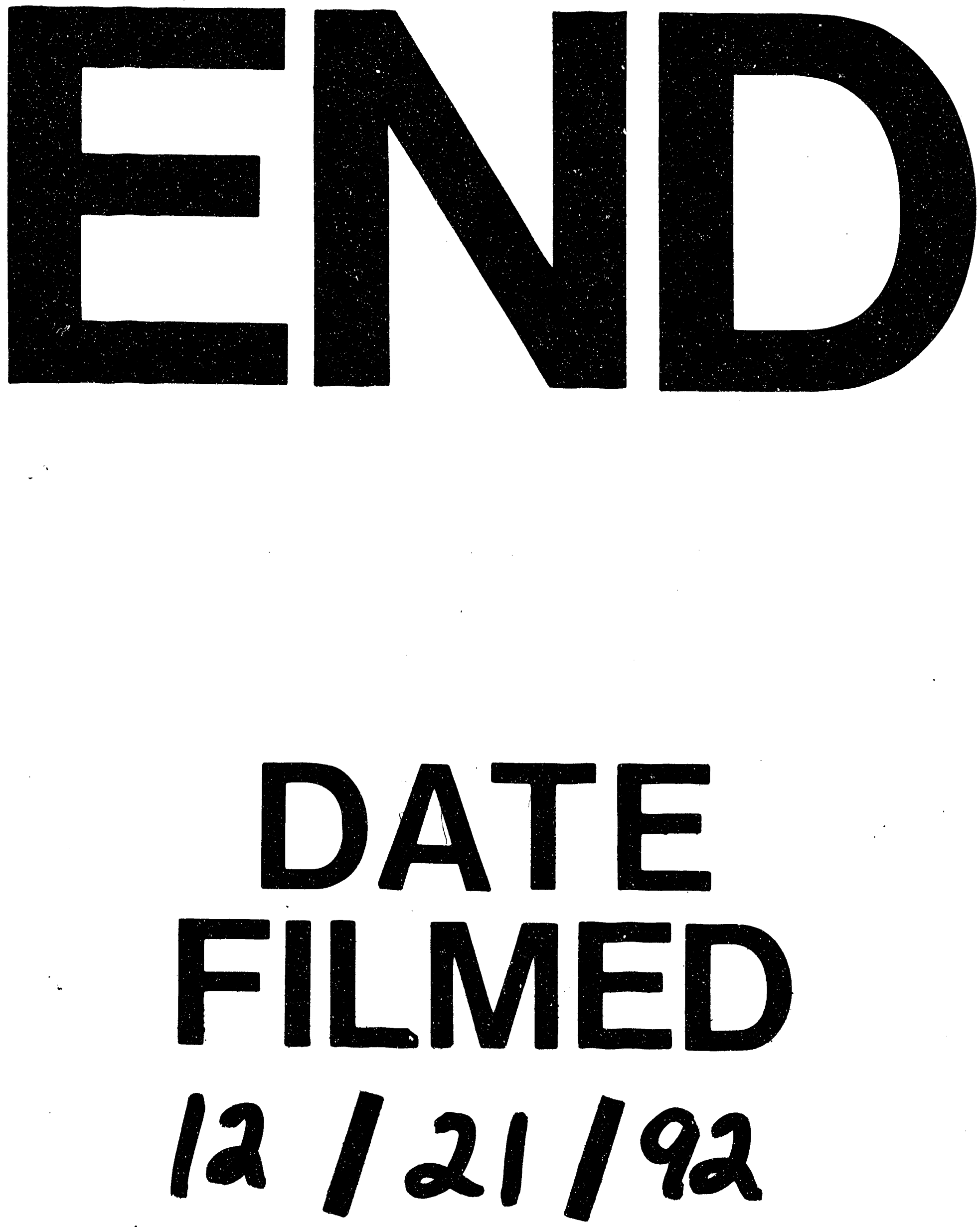